\title{
Implementación de sistema de control automático de temperatura en proceso de parafinado mediante LabVIEW
}

\section{Implementation of automatic temperature control system in the process of paraffining by LabVIEW}

\author{
SÁNCHEZ-LÓPEZ, Héctor Javier†**, ROJAS-OLMEDO, Israel Alejandro, GONZÁLEZ- \\ GOMEZTAGLE, Aldo y ROJAS-RAMÍREZ, Erick
}

Universidad Tecnológica del valle de Toluca, C. del Depto. del D.F. km 7.5, Santa María Atarasquillo, Lerma, México

ID $1^{\text {er }}$ Autor: Héctor Javier, Sánchez-López

ID $1{ }^{\mathrm{er}}$ Coautor: Israel Alejandro, Rojas-Olmedo

ID $2^{\text {do }}$ Coautor: Aldo, González- Gomeztagle

ID $3^{\text {er }}$ Coautor: Erick, Rojas-Ramírez

DOI: $10.35429 / J E A .2019 .21 .6 .10 .13$

Recibido: 19 de Agosto 2019; Aceptado 30 Noviembre, 2019

\begin{abstract}
Resumen
El presente proyecto se enfoca en procesos de automatización para el control de variables de temperatura y longitud debido a la necesidad de controlar algunas de las principales variables físicas que forman parte del proceso de producción|, con ello obtener las señales físicas e interpretarlas para su procesamiento y asignación de una valoración de referencia medible, que permita controlar los valores para determinar el comportamiento de los procesos. Dichos procesos de automatización se desarrollaron mediante control asistido por computadora, software de programación grafica LabVIEW y una tarjeta de adquisición de datos USB NI 6000. Los procesos por implementar están basados en: el control y monitoreo de temperatura en el área de pabileras, control y monitoreo de proporción en el mixtado de los cerillos, así como el control de tamaño y temperatura en la producción del pabilo. Estos procesos de automatización para el control de variables de calidad permiten manipular y monitorear las variables físicas como temperatura y longitud, ya que influyen en gran medida para la producción de cerillos. Permitiendo al usuario realizar ajustes a los valores deseados en el área de producción y mantenerlos lo más estables con referencia a los estándares de calidad. Se muestra la implementación de un sistema de control automático de temperatura para el proceso de parafina mediante LabVIEW, se realizará un análisis en el área de producción de pábilo y parafina de la empresa Cerillera la Central S.A. de C.V. en el cual se debe controlará la temperatura de $79^{\circ} \mathrm{C}$ a $87{ }^{\circ} \mathrm{C}$, mediante electroválvulas activadas por relevadores a $127 \mathrm{~V}$ C.A, se empleará un sensor resistivo (PT-100) acoplado a un puente Wheatstone el cual entregara datos analógicos; los cuales serán procesados por la tarjeta de adquisición de datos NI USB-6000, una PC y software NI- LabVIEW con el objetivo de encender o apagar las electroválvulas que activan el suministran de vapor para el calentamiento de parafina.
\end{abstract}

Tarjeta de adquisición de datos USB NI 6000, Pabileras, Mixtado

\begin{abstract}
This paper details the design the automation processes for the control of temperature and length variables due to the need to control some of the main physical variables that are part of the production process |, thereby obtaining the physical signals and interpreting them for processing and assignment of a measurable baseline assessment, which allows controlling the values to determine the behavior of the processes. These automation processes were developed using computer-assisted control, LabVIEW graphic programming software and an NI 6000 USB data acquisition card. The processes to be implemented are based on: the control and monitoring of temperature in the area of pabileras, control and monitoring of proportion in the mixing of matches, as well as the control of size and temperature in the production of the wick. These automation processes for the control of quality variables allow the manipulation and monitoring of physical variables such as temperature and length, since they greatly influence the production of matches. Allowing the user to adjust the desired values in the production area and keep them as stable with reference to quality standards. The implementation of an automatic temperature control system for the paraffin process through LabVIEW is shown, an analysis will be carried out in the area of pábilo and paraffin production of the company Cerillera la Central S.A. de C.V. in which the temperature of $174^{\circ} \mathrm{F}$ to $188.6^{\circ}$ F must be controlled, by means of solenoid valves activated by relays at 127 VAC, a resistive sensor (PT-100) coupled to a Wheatstone bridge will be used which will deliver analog data; which will be processed by the NI USB-6000 data acquisition card, a PC and NILabVIEW software in order to turn on or off the solenoid valves that activate the steam supply for paraffin heating.
\end{abstract}

NI 6000 USB data acquisition card, Pabileras, Mixing

Citación: SÁNCHEZ-LÓPEZ, Héctor Javier, ROJAS-OLMEDO, Israel Alejandro, GONZÁLEZ- GOMEZTAGLE, Aldo y ROJAS-RAMÍREZ, Erick. Implementación de sistema de control automático de temperatura en proceso de parafinado mediante LabVIEW. Revista de Aplicaciones de la Ingeniería. 2019. 6-21: 10-13

\footnotetext{
* Correspondencia del Autor (Correo electrónico: hector.sanchez@utvtol.edu.mx)

$\dagger$ Investigador contribuyendo como primer autor.
} 


\section{Introducción}

Se implementa un sistema de control automático de temperatura para el proceso de parafina mediante LabVIEW, se realizará un análisis en el área de producción de pábilo y parafina de la empresa Cerillera la Central S.A. de C.V. en el cual se debe controlará la temperatura de $79{ }^{\circ} \mathrm{C}$ a $87{ }^{\circ} \mathrm{C}$, mediante electroválvulas activadas por relevadores a $127 \mathrm{~V} \mathrm{C.A,} \mathrm{se} \mathrm{empleará} \mathrm{un} \mathrm{sensor}$ resistivo (PT-100) acoplado a un puente Wheatstone el cual entregara datos analógicos; los cuales serán procesados por la tarjeta de adquisición de datos NI USB-6000, una PC y software NI- LabVIEW con el objetivo de encender o apagar las electroválvulas que activan el suministran de vapor para el calentamiento de parafina.

\section{Control de temperatura para el depósito de la parafina}

La señal de termopar se adquiere mediante la tarjeta de adquisición de datos. Se monitorea, visualiza, controla y se almacena la temperatura del depósito de la parafina mediante el software de programación gráfico de LabVIEW el diagrama a bloques del control se muestra en la figura 1 .

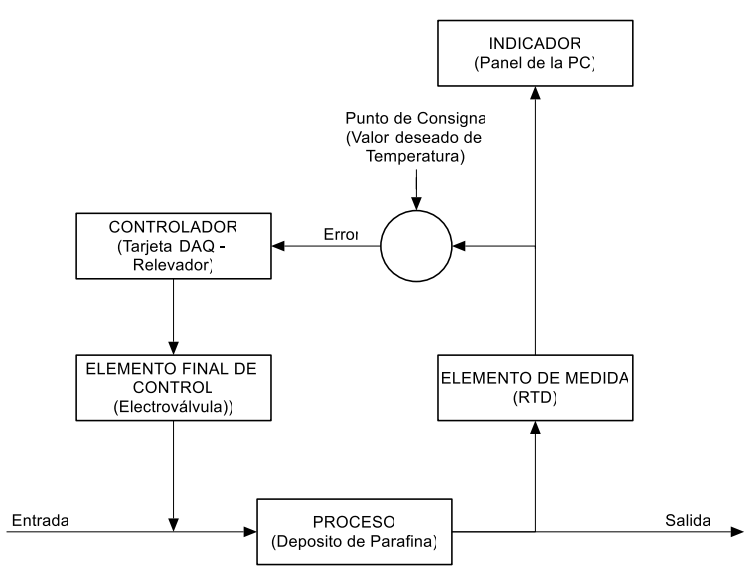

Figura 1 Diagrama a bloques para el control de temperatura para el depósito de la parafina

\section{Máquina de estados}

Para el control y monitoreo de la temperatura del depósito de la parafina se diseñó la máquina de estados que se muestra en la figura 2. La cual consta de cuatro estados S0, S1, S2 y S3 enlistados en la tabla 1. Esta máquina está diseñada para identificar cuando ocurre un evento, es decir, cuando se accionan los controles del panel frontal del instrumento virtual y de esta manera tomar decisiones especificas en el programa.
Dichas condiciones partirán del estado inicial (S0) al comienzo del programa y según ocurran los eventos en los controles Botón ON, Botón OFF y Botón ATOMÁTICO, los estados cambiarán de un estado presente a un estado futuro.

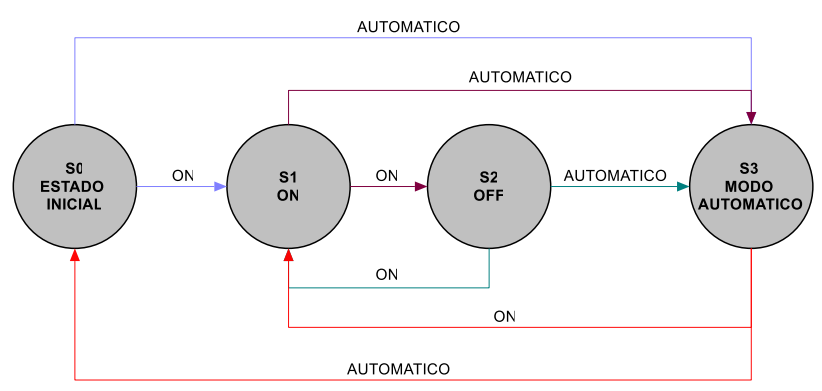

Figura 2 Máquina de estados para el control y monitoreo de la temperatura

\begin{tabular}{|l|l|l|}
\hline Estado Actual & \multicolumn{2}{c|}{ Estado Futuro } \\
& Boton On & \multicolumn{1}{c|}{ Boton Automatico } \\
\hline S0-ESTADO INICIAL & S1 & S3 \\
\hline S1-ON & S2 & S3 \\
\hline S2-OFF & S1 & S3 \\
\hline S3-AUTOMATICO & S1 & S0 \\
\hline
\end{tabular}

Tabla 1 Estados para control y monitoreo de la temperatura

\section{Desarrollo y diseño de programación para el control del proceso con LabVIEW}

El desarrollo del monitoreo y control de temperatura consta de tres subprogramas principales:

\footnotetext{
- Intervalos de control para temperatura de señal del termopar.

- Máquinas de estado.

- $\quad$ Registro de datos.
}

\section{Intervalos de control para temperatura de señal del termopar}

Los intervalos de control para la temperatura serán ajustables según lo requiera el proceso, así mismo cumplirán con tres condiciones para que opere el elemento de control, dichas condicionantes tendrán efecto de control cuando la temperatura se encuentre baja, adecuada y alta (ver figura 3). 


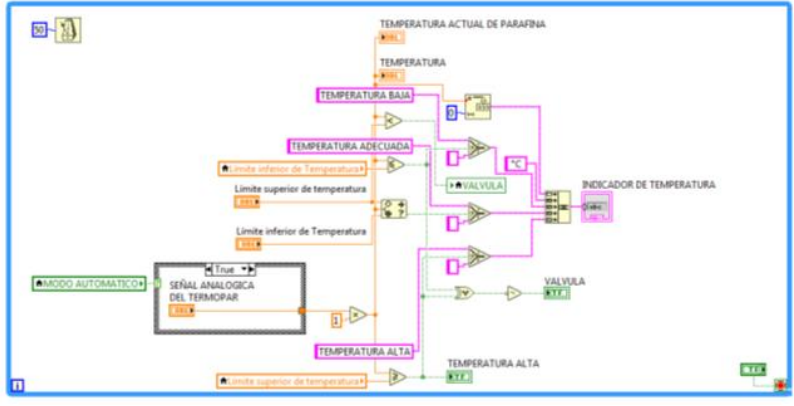

Figura 3 Intervalos de control

\section{Máquinas de estado}

La máquina de estados está diseñada para identificar cuando ocurre un evento, esto quiere decir, cuando un control del panel frontal del instrumento virtual sea accionado, LabVIEW mediante la estructura de eventos identificará el evento para cumplir la función asignada ver figura 4.

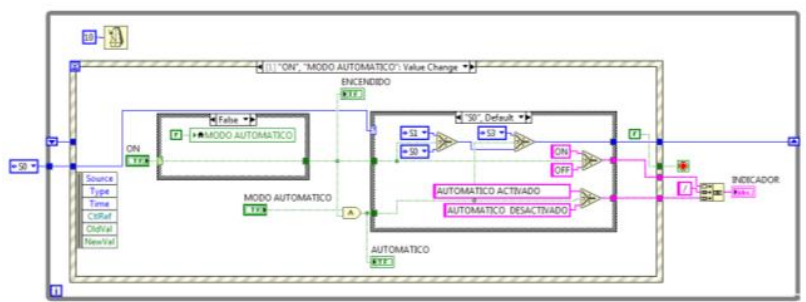

Figura 4 Máquina de estado mediante la estructura de eventos

\section{Registro de datos}

Este apartado del programa realiza el registro de datos de los valores de temperatura del depósito de la parafina y la temperatura del agua leídos por el sensor y además cuenta con la facilidad de exportación de estos a Microsoft Excel ver figura 5.

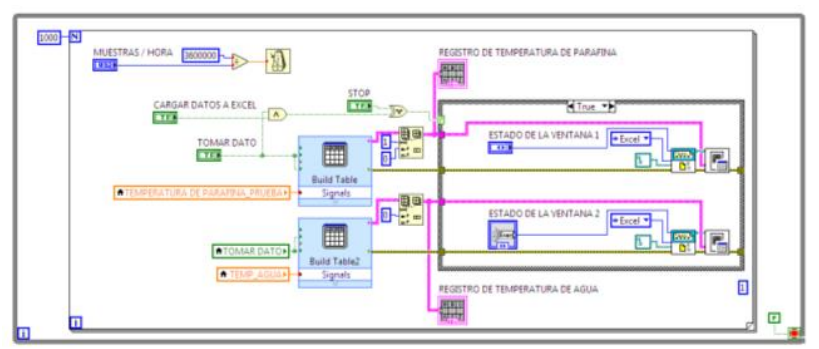

Figura 5 Registro y exportación de datos

\section{Diseño del panel frontal}

Para el control, visualización y almacenamiento de la información el instrumento virtual cuenta con panel frontal que contiene controles e indicadores.
Los controles son: encendido, regulación automática, retardo del accionamiento de la válvula de control, inicio del almacenamiento de datos, carga de datos almacenados en Excel, minimizar o maximizar las ventanas del registro de datos y paro.

Los indicadores son: gráfico para visualizar el comportamiento de la temperatura de la parafina, termómetro para visualizar la temperatura actual de la parafina, termómetro para visualizar la temperatura del agua y dos indicadores de tipo cadena donde se visualiza los datos almacenados de las temperaturas de la parafina y del agua registrados por fecha y hora ver figura 6.

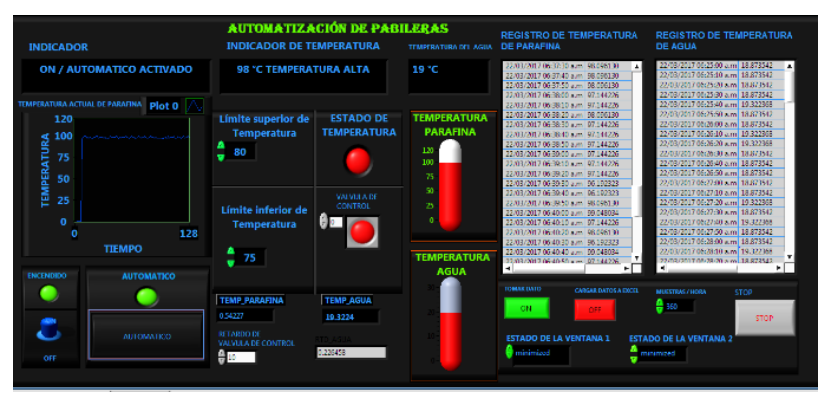

Figura 6 Panel de control

\section{Resultados}

La implementación del proyecto se realizó en el área de pabilo, con el objetivo de mejorar las propiedades de éste, de tal manera que el producto mejore sus características físicas para procesos posteriores. El instrumento virtual programado junto con la tarjeta de adquisición de datos, el termopar y la válvula permitieron controlar el proceso de parafinado del pabilo en tiempo real, regulando las variaciones de temperatura, manteniéndolas dentro del intervalo de 80 a $90{ }^{\circ} \mathrm{C}$.

En la figura 7 se muestra el sistema implementado. El cual consta de la tarjeta NI USB 6000, para accionar la apertura o cierre de la electroválvula se utilizaron dos relevadores a $5 \mathrm{~V}$ que son accionados por el controlador L293D que recibe las señales de la tarjeta de adquisición de datos.

Para la alimentación de la electroválvula y los relevadores se utilizaron dos fuentes de alimentación de 24 VCD y 5VCD respectivamente. 


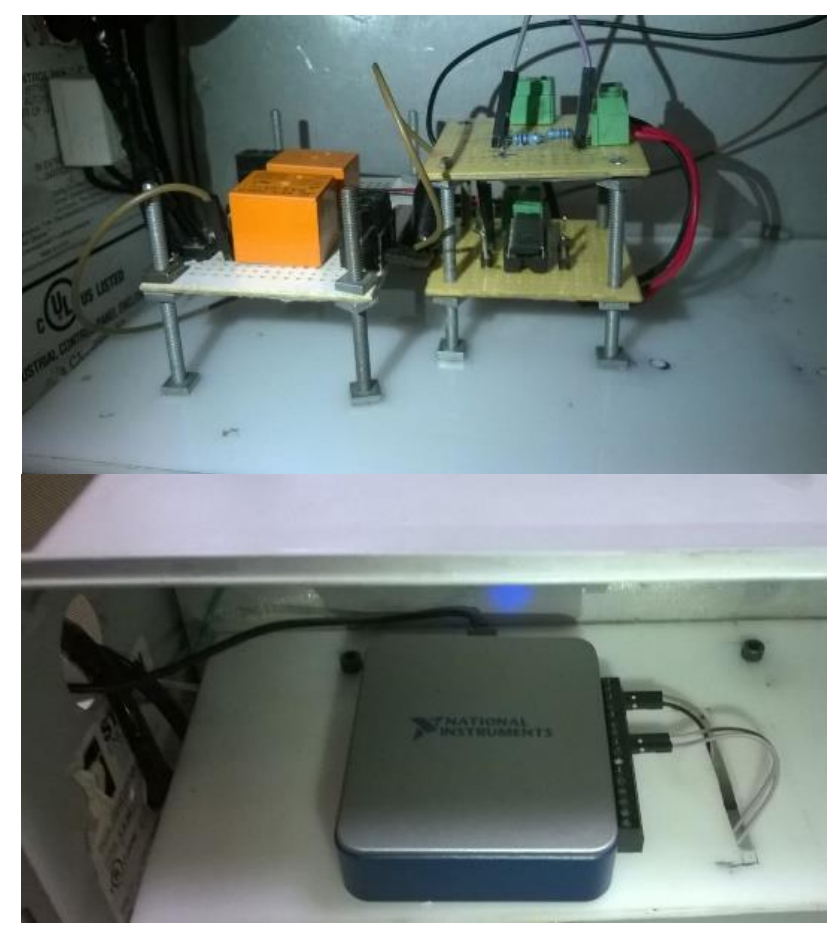

Figura 7 Implementación del sistema

El registro de datos en el proyecto es importante, para generar estadística del comportamiento de la temperatura en el proceso y determinar si el proceso se mantiene estable en las figuras 8 y 9 se muestran las gráficas del comportamiento de la temperatura de la parafina y del agua respectivamente. En done se puede observar la variación de la temperatura en un intervalo de 80 a $90{ }^{\circ} \mathrm{C}$ para la parafina mientras que la temperatura en el agua se mantiene a 20 ${ }^{\circ} \mathrm{C}$.

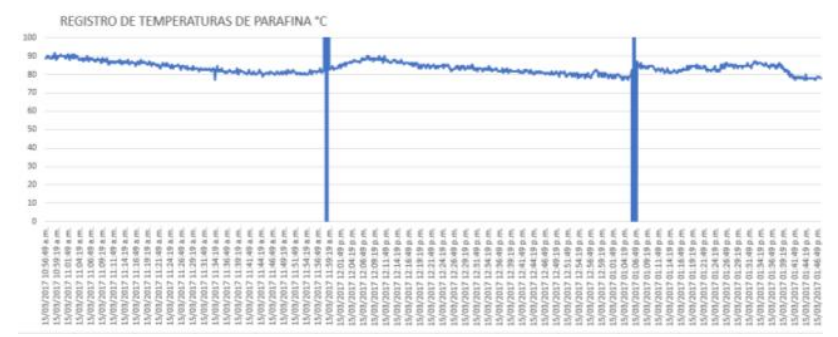

Figura 8 Registro de temperaturas de parafina

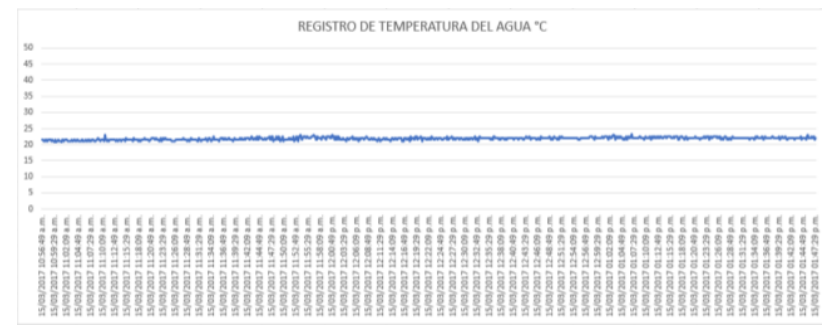

Figura 9 Registro de temperaturas del agua

\section{Agradecimiento}

Los autores agradecen a la Universidad Tecnológica del Valle de Toluca, así como a la empresa cerillera La Central S.A de C.V. el desarrollo del presente trabajo.

\section{Conclusiones}

La implementación del proyecto reflejó en base a la adquisición de datos e información enviada se logra establecer un control y monitoreo del proceso productivo mediante el software LabVIEW para generar estadísticas de control de variables de calidad, que serán analizados por el departamento de calidad de la empresa para fines propios de la misma además se facilita el diseño y desarrollo de la automatización de sistemas productivos, la mejorar la calidad del producto, la seguridad para evitar lesiones al personal, se redujo el desperdicio y los tiempos muertos del proceso. como propuesta futura se puede activar o desactivar vía remota desde FluidSim.

\section{Referencias}

Coughlin R. C. (1993.). Amplificadores operacionales y circuitos integrados lineales. PEARSON.

Cruz A. S. (2011). Instrumentación Industrial (Octava ed.). Alfaomega.

Floyd T. L. (2008). Dispositivos Electrónicos. PEARSON EDUCACIÓN.

Larsen R. W. (2014). LabVIEW para Ingenieros. México: PEARSON.

Ogata, K. (2010). Ingeniería de control moderna (Quinta ed.). PEARSON EDUCACIÓN S.A. 